МОДЕЛИ МЕТЕОРОЛОГИЧЕСКИХ ПОКАЗАТЕЛЕЙ ДЛЯ АНАЛИЗА e-mail: pgs@angtu.ru

\title{
ТЕПЛОПОТРЕБЛЕНИЯ ЗДАНИЙ
}

Kuzmin S.I., Zelenina A.A.

\section{MODELS OF METEOROLOGICAL INDICATORS FOR ANALYSIS OF BUILDING HEAT CONSUMPTION}

\begin{abstract}
Аннотация. Предложена модель метеорологических показателей наружного климата для определения теплопотребления системы отопления.

Ключевые слова: расчетная температура наружного воздуха, система отопления, теплоотдача, гидравлическая характеристика, приведенные затраты.

Abstract. A model of meteorological indicators of the external climate to determine the heat consumption of the heating system has been proposed.

Keywords: estimated outdoor air temperature, heating system, heat output, hydraulic charac-
\end{abstract} teristic, given costs.

Для выбора мощности и анализа работы теплогенерирующего оборудования системы отопления здания важно знать затраты тепла в некоторый произвольный отрезок отопительного периода $W_{O . i}$ (Втчас), которые зависят от климатических параметров района строительства [1]:

$$
W_{O . i}=Q_{c o} \sum_{i=10}^{t_{H .0}} \frac{t_{B}-t_{t . i}}{t_{B}-t_{H .0}} \cdot \tau_{n i}
$$

где $Q_{c o}$ - расчетная мощность системы отопления здания, Вт; $t_{b}, t_{H .0} n t_{t . i}$ - температуры соответственно внутреннего воздуха, наружного по параметрам категории «Б» и средняя за некоторый отрезок отопительного периода, ${ }^{\circ} \mathrm{C}$;

$\tau_{t i}$ - продолжительность отрезка отопительного периода, в котором наблюдается температура воздуха $t_{t . i}$, час.

Точность результатов, получаемых из выражения (1) тем выше, чем меньше отрезок $\tau_{t i}$. Однако использование уравнения (1) для небольших по продолжительности отрезков затруднительно, так как необходимо использовать частные статистические характеристики климата, имеющие существенные отличия по географическому положению объекта. Поэтому представляется полезным выразить составляющую $\sum_{i=10}^{t_{H .0}} \frac{t_{s}-t_{t . i}}{t_{s}-t_{H .0}} * \tau_{n i}$ в (1), в виде функциональной 
зависимости от определяющего параметра - текущей температуры наружного воздуха.

Распределение продолжительности периодов стояния температур наружного воздуха в течение среднестатистического года приводятся в [2] для представительских пунктов основных климатических зон страны. На рис 1 приведены графики распределения часов стояния температур для пунктов «холодного» - Салехард, «умеренно-холодного» - Улан-Удэ, «умеренного» - Москва и «умеренно-теплого с мягкой зимой» - Новороссийск в диапазоне температур отопительного периода (от $+10^{\circ} \mathrm{C}$ и ниже).

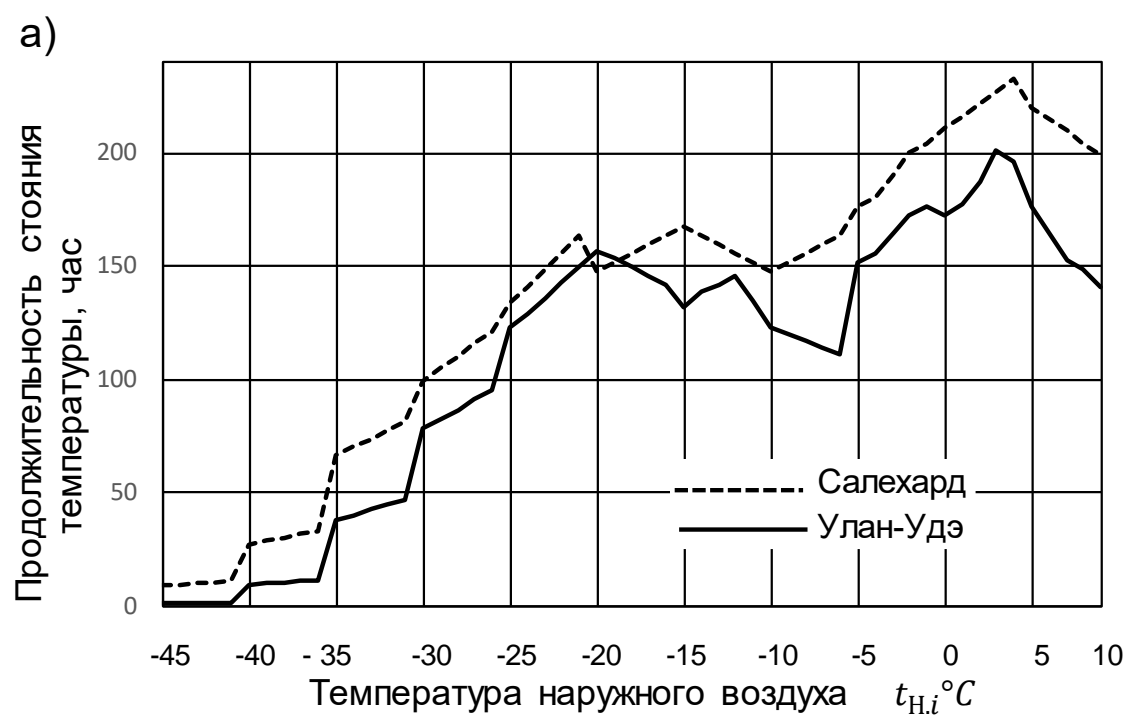

б)

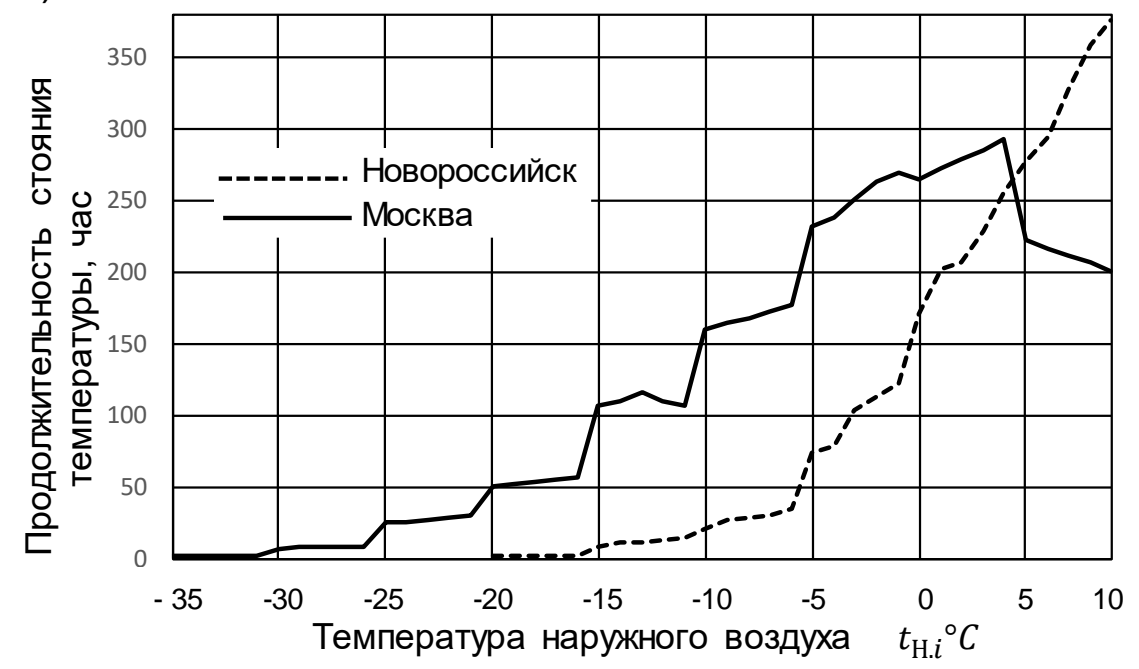

Рисунок 1 - Продолжительность стояния температуры наружного воздуха для пунктов: а) - «холодного» и «умеренно-холодного», б) - «умеренного» и «умеренно-теплого» 
Как следует из графиков, продолжительности стояния температур сложно представить в виде функциональной зависимости. Однако, если выразить распределение параметров через интегральный показатель:

$$
k_{t \tau . i}=\sum_{i=10}^{t_{H .0}} \frac{t_{\sigma}-t_{t . i}}{t_{\sigma}-t_{H .0}} \bullet \tau_{n i}=f\left(\Delta t_{H i}\right),
$$

то, проявляется определенная «гладкая» зависимость (рис. 2), которую можно интерпретировать через приближенное уравнение $f\left(\Delta t_{H i}\right)$.

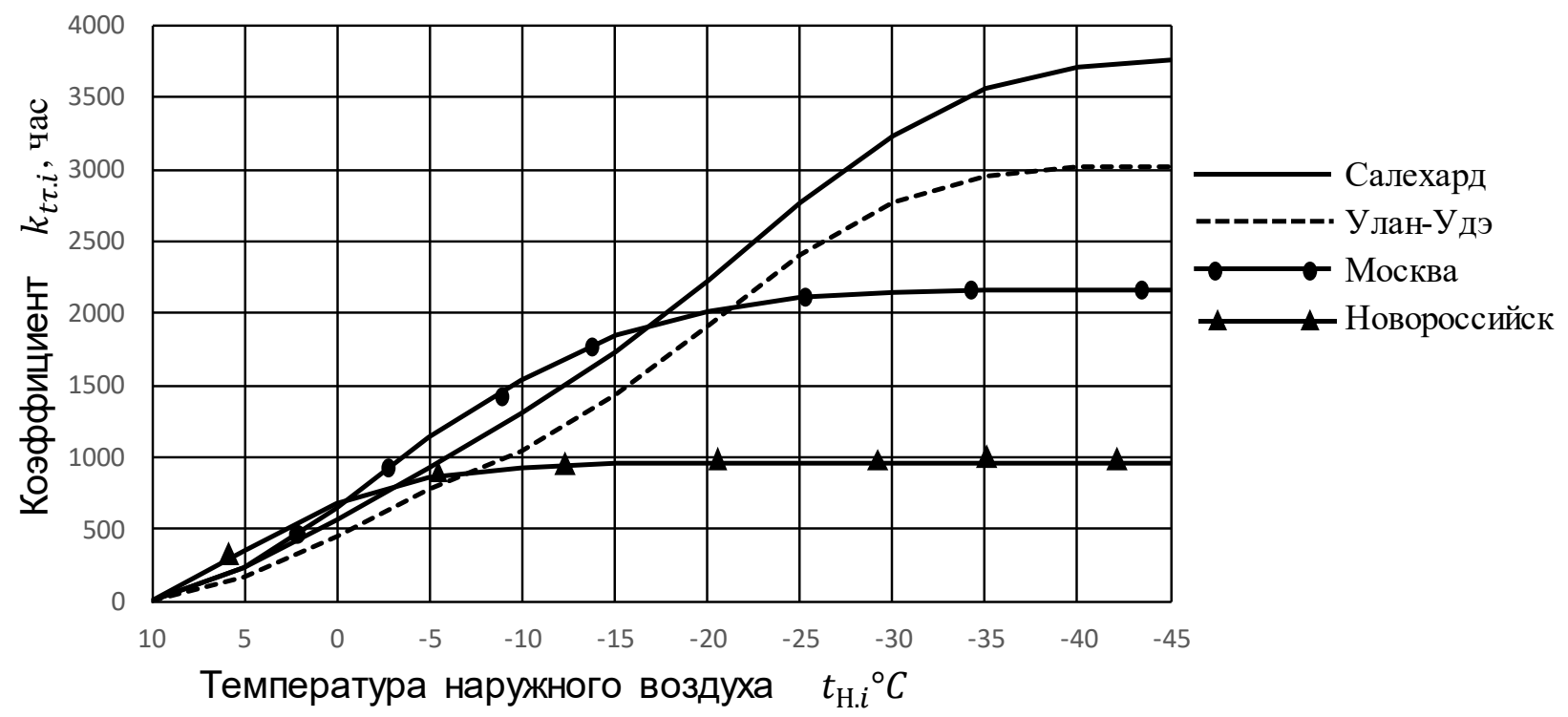

Рисунок 2 - Интегральная продолжительность стояния температуры наружного воздуха

Таким образом получаем следующие уравнения приближенной регрессии для выбранных географических пунктов:

- Салехард: $k_{t \tau . i}=495+0,04 \cdot t_{H . i}^{3}+1,96 \cdot t_{H . i}^{2}-68,05 \cdot t_{H . i}$

- Улан-Удэ: $k_{t \tau . i}=380+0,0425 \cdot t_{H . i}^{3}+1,99 \cdot t_{H . i}^{2}-57,23 \cdot t_{H . i}$

- Москва: $k_{t \tau . i}=770+0,037 \cdot t_{H . i}^{3}-0,95 \cdot t_{H . i}^{2}-80 \cdot t_{H . i}$

- Новороссийск: $k_{t \tau . i}=643+0,0214 \cdot t_{H . i}^{3}-1,805 \cdot t_{H . i}^{2}-49,45 \cdot t_{H . i}$

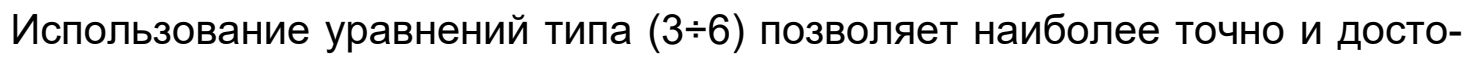
верно определять теплопотребление системы отопления зданий, но при условии известного статистического распределения периодов стояния температуры, так как даже в одной климатической зоне эти распределения не универсальны и не обладают признаками подобия.

Для более «грубого» определения теплопотребления за отопительный период в целом используется выражение (7): 


$$
W_{\text {O.П }}=Q_{c o} \frac{t_{B}-t_{o n}}{t_{B}-t_{H}} * \tau_{o n} * 24
$$

где $t_{H}$ и $t_{\text {on }}$ - температуры соответственно наружного воздуха по параметрам категории «Б» и средняя за отопительный период, ${ }^{\circ} \mathrm{C} ; \tau_{\text {on }}$ - продолжительность отопительного периода, сутки.

Анализ выражения (1) затрудняет наличие большого количества показателей и их произвольное значение. Поэтому представляется полезным выявить зависимости между климатическими параметрами, входящими в фрормулу (7), с целью ее упрощения.

Произведение разности температур $\left(t_{6}-t_{o n}\right)$ и продолжительности отопительного периода $\tau_{\text {on }}$ представляет собой «градусо-сутки» отопительного периода - $Z_{\text {оn }}[4]$ :

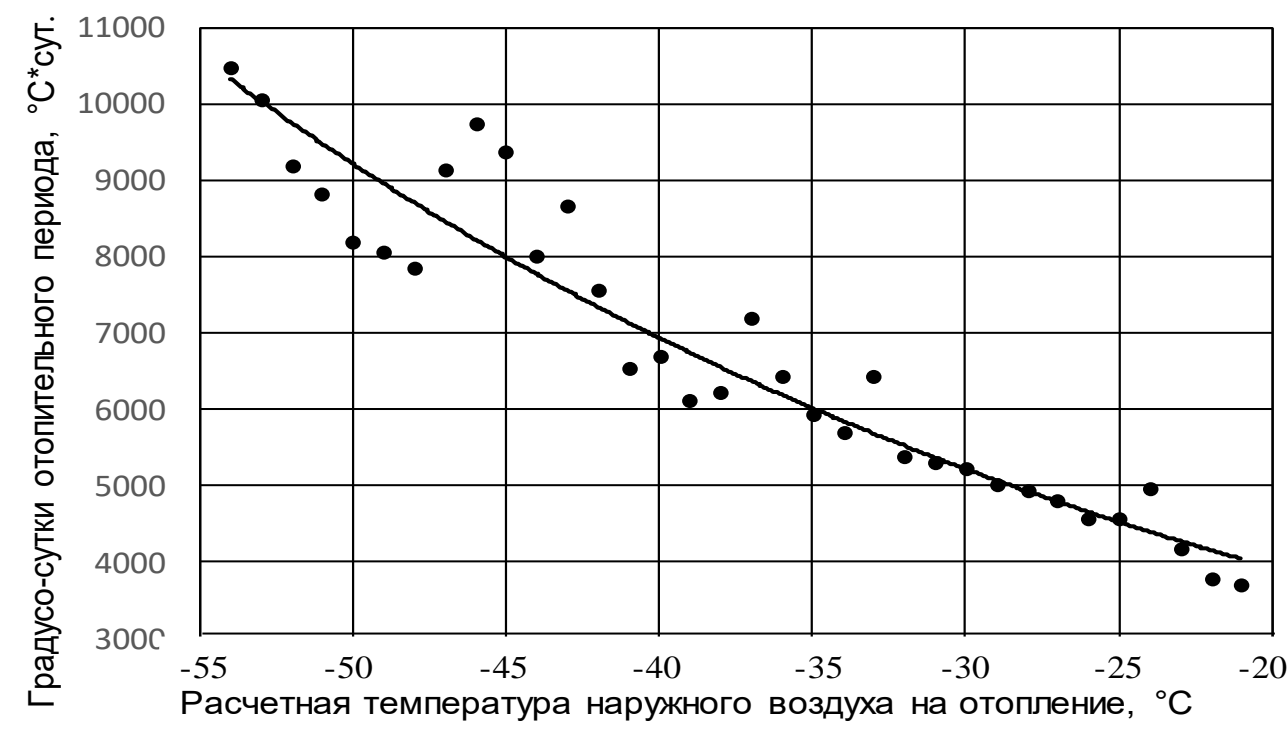

Рисунок 3 - Распределение «градусо-суток» от расчетной температуры наружного воздуха по параметрам категории «Б»

На рисунке 3 представлены данные, выражающие стохастическую связь между расчетной температурой наружного воздуха по параметрам категории «Б» и «градусо-сутками» отопительного периода для географических пунктов климатических районов при $t_{8}=20^{\circ} \mathrm{C}$ [3].

Принимая значения $Z_{\text {on }}$, представленные на рисунке 3 в качестве «облака» статистических данных, получено следующее уравнение регрессии, описывающие соответствующее распределение с 12 \% точностью:

$$
Z_{\text {on }}=2280 \cdot \exp \left(-0,028 \cdot t_{H}\right)
$$


Таким образом, уравнение (7) можно представить как функциональную зависимость только от одной переменной - $t_{\mu}^{5}$ :

$$
W_{\text {O.П }}=Q_{c o} \frac{24720 \cdot \exp \left(-0,028 \cdot t_{H}\right)}{t_{B}-t_{H}}
$$

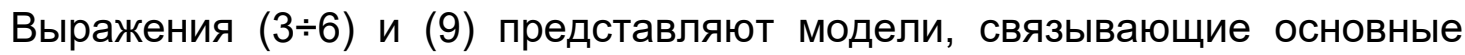
метеорологические характеристики населенного пункта, и могут служить основой для анализа теплопотребления систем отопления зданий и эффективности энергосберегающих мероприятий.

\section{ЛИТЕРАТУРА}

1. СП 60.13330. Отопление, вентиляция, кондиционирование.

2. ГОСТ 16350-80 Климат СССР.

3. Справочник проектировщика. Внутренние санитарно- технические системы // Под. Ред. И.Г. Староверова ч.1 Отопление. - М., Стройиздат, 1993.- 896 с.

4. СП 131.13330.2018 Строительная климатология.

5. СП 50.13330.2012 Тепловая защита зданий. 